aponeurotic expansion of the transversalis is covered only by the latissimus. Lesshaft calls this the "trigonum lumbale superius," to distinguish it from Petit's triangle, the trigonum lumbale inferius, and thinks, with Grynfeltt, that it may very well serve for the exit of a lumbar hernia. Though later writers have questioned whether a hernia ever came through this space, the case of Mr. J. Hutchinson, jun., apparently disposes of the doubt. ${ }^{18}$ For his hernia came through an aperture one inch and a half to two inches above the iliac crest and near the last rib. He says the protrusion in his case was "through the transversalis aponeurosis and the latissimus dorsi, where the latter arises from the strong fascia covering the erector spinæ," thus leaving it doubtful whether the latissimus was muscular or tendinous at its line of contact with the neck of the sac. Dr. Hume ${ }^{19}$ was unfortunately not permitted to make a post-mortem examination in his case ; but from the description it is probable that the hernia escaped by this route, and the latissimus dorsi was thought partly to cover the tumour. The upper triangle is more constant than "Petit's, which is frequently absent either because the latissimus dorsi overlaps the external oblique, or because the borders of these muscles are contiguous. Lesshaft examined 108 adult and thirty-five infant corpses, and found the triangle of Petit present in 77 per cent. of the adult and in 25 per cent. of the children. It is obvious, then, that Petit's triangle oftentimes develops as the growth of the body proceeds, and this in some measure explains why elderly adults, and not children, are principally affected with lumbar hernia. The floor of this lower triangle is covered by a stout fascia, beneath which are the muscular fibres of the internal oblique, and deeper still the aponeurosis of the transversalis abdominis. In some cases, according to Lesshaft, ${ }^{20}$ the internal oblique at this spot is wholly tendinous. It may be, then, that hernia through this triangle are confined to the cases where fibrous, and not muscular, tissues occlude the opening. Prof. Braun, after making a dissection of a case which followed a spinal abscess, supposes that lumbar hernia may escape by the apertures for the cutaneous nerves. A branch from the posterior lumbar nerves pierces the abdominal wall close to the triangle of Petit, so close indeed that the two gaps almost coalesce. This is a revival of the suggestion whereby Sir Astley Cooper sought to explain the origin of ventral hernia, to which it was long since objected that the nerves run between the muscles, and do not penetrate the whole thickness of the parietes at one spot; and the objection is still at hand to condemn once more this notion now adopted by the professor.

The cases of spontaneous lumbar hernia, presented in the tables above referred to with those just related, amount to twenty-five, but many are defective in important particulars. In eleven the protrusion is stated to have occurred at the triangle of Petit, in two it appears to have come by the upper lumbar triangle, in one, to be presently described, near the tip of the twelfth rib, and in others the position of the opening is not clearly defined. The two cases given by Coze, which are quoted by Braun, ${ }^{21}$ offer a number of extraordinary coincidences. Both were artillery men, and they were affected about the same time; in both a muscle hernia appeared at the right triangle of Petit, and, after an interval of three months, in both an intestinal hernia occurred. The recital of facts so strange and rare, happening twice over at the same time, calls up an uncontrollable feeling of insecurity.

$$
\text { (To be concluded.) }
$$

18 London Hospital Museum, preparation 1320 Brit. Med. Jour., July 13th, 1889

20 Loc. cit., $271 . \quad 21$ Loc. cit., pp. $212,213$.

Bridgwater Infirmary. - Mr. F. Valiant of Cossington (president for the year) occupied the chair at the seventy-seventh annual meeting, held on Oct. 15th. The efficiency of the institution has been fully sustained. During the year, 235 in-patients were admitted; the out-patients numbered 1493, and the dental cases 101. The finances of the charity had, on the whole, been well maintained. The reserved fund amounted to $£ 5350$. The report alluded to the resignation of Mr. John Parsons as senior medical officer, after holding that office for nearly forty-five years, and a resolution was carried to place a brass tablet in the entrance hall of the infirmary, with a suitable inscription to commemorate his services.

\section{THE ANILINE DYES AS ANTISEPTICS, AND THEIR USE IN PRACTICE'.}

Bx J. STILLING, M.D.,

PROYESSOR IN THE UNIVERSITY OF STRASSBURG.

Translated and much Condensed by Edgar Stevenson, M.B.

IN spite of all the important theoretical changes that modern bacteriology has produced in medical science, it still leaves much to be desired so far as practical application of its teachings is concerned. The fact that the existing antiseptics are far from perfect induced me to make a series of experiments on the aniline bodies with a view to finding out their antiseptic properties. A perfect antiseptic ought to be a substance which is able to destroy bacteria, and at the same time be harmless to the human organism. It must also be very diffusible, not only as regards its action on the tissues, but also so as to be able to spread through a whole colony of micro-organisms. I found, after some years' work, that certain aniline dyes, especially the violet ones, possess all these required properties, and I shall now proceed to give briefly the results of some of my experiments.

1. Botanical and bacteriological experiments (carried out in conjunction with Dr. J. Wortmann).- Preliminary experiments showed us that the violet dyes especially possessed in a high degree the faculty of preventing the development of bacteria. For the sake of brevity I shall use the term "methyl violet" for the whole series of these violet dyes. Paste made with wheaten meal, and a 2 per 1000 solution of methyl violet added, does not turn suar however long it is kept. Milk with $m$. va added does not turn sour, and butter and bacon fat treated with $m$. v. do not turn rancid. To test its antiseptic properties, however, we decided to use moulds and the bacteria of putrefaction, as being more resistant than pathogenic micro-organisms, and of all moulds the mucor stolonifer was chosen on account of its very rapid growth and great resistance. The mucor stolonifer was sown upon small rolls of bread, some of which were soaked in a 1-500 and 1-1000 solution of $\mathrm{m} . \mathrm{v}$., some in water only. On the latter a full and luxuriant growth appeared in twenty-four hours, whilst on the former there was no sign of growth, even after fourteen days. In another experiment, mixed moulds were sown on sugar meat-extract, which was then brushed in streaks and spots with a $\frac{1}{2}$ per cent. solution of $m$. $v$. As soon as a growth of mycelium appeared, it began to spread out from its centre until it reached one of the spots or streaks, when its further progress was immediately stopped. Other experiments confirmatory of this were made with varying strengths of $m$. v. solution, and under varying conditions. Similar resuits to the above were obtained, except in very weak solutions $(1-25000)$ where a weak growth of mould appeared, getting stronger as the strength of the solutions decreased. Experiments were made on the bacteria of putrefaction in the following way: Solutions of $m$. v. of strengths of $1-1000$ to $1-64,000$ were taken, and 60 c. c. of each poured into small flasks. In each of these flasks was placed a small piece of raw beef, the flasks being then closed with cotton-wool, and all placed in the hot chamber at a temperature of $25^{\circ} \mathrm{C}$. Every twenty-fou hours each medium was carefully searched under the microscope for bacteria of putrefaction. The control glass, which had been treated with water alone, at the same time as the others, showed in twenty-four hours stinking putrefaction. On the sixth day there was no sign of putrefaction in solutions up to $1-4000$. Solutions of $1-64,000$ in some cases showed signs of commencing putrefaction after a twentyfour hours' exposure; and in some cases also those of a strength of 1-40,000 showed moderate colonies of bacteria, but in these cases the bacteria were partly stained blue, so that it was quite evident that their growth and development were greatly retarded.

From this it appears that $m$. v. in suitable strengths acts as a complete check to the development of bacteria of putrefaction; and as they are more resistant than pathogenic organisms, it must be taken for granted that the latter would very easily succumb to their action. This was confirmed by direct experiment with staphylococcus pyogenes aureus. Besides the violet dyes we tried a number of other 
dyes, such as fuchsin, methyl blue, rhodamin vesuvin, and many others. The lest of these proved to be ethyl violet, but the difficulty in obtaining it absolntely pure forms an objection to its use. To methyl violet $I$ have given the name "pyoctanin," and it can be obtained absolutely pure from the well-known firm of E. Merck in Darmstadt. Certain auramines proved to be the next best, when used in solutions of $1-4000$ to $1-1000$.

We next tried the action of $\mathrm{m}$. $\mathrm{v}$. on the enzymes, and as a typical example for this purpose we took the rliastatic ferment of malt. In solutions of 1-1000 and 1-2000 a striking retardation of its diastatic action on starch granules was observed.

2. Experiments on animals-As my original purpose in heginning these experiments was to find an antiseptic for ophthalmic use, I began by experimenting on rabbits' eyes, and for this purpose of course chose albinos. A 1-1000 solution dropped into the eye stains the conjunctiva, sclerotic, and iris, but not the cornea, if it be unwounded. The staining passes off by the next day. If $\mathrm{m}$. $\mathrm{v}$. in bulk be powdered in to the conjunctival sac, it is all stained very deeply, and here and there the substance precipitates on the conjunctiva and cornea with a metallic lustre. In a day or two the epithelium is all shed off in large flakes, which show under the microscope a striking fatty degeneration, almost like that of the phosphorous kidney; but, nevertheless, these flakes, as well as the whole conjunctival sac are quite free from bacteria. The appearance of the staining is the same as in other mucous tissues. There is a peculiar action of $m$. $v$, which often takes places in the human eye-viz., a great dilatation of the pupil without any paralysis of accommodation. Subcutaneous injection of $: 0$ centimetres or more of a 1-1000 solution will not cause bad effects in rabbits or guinea-pigs, and even comparatively large quanties ( 10 centimetres) can be injected in to the peritoneal cavity without morbid appearances, but larger quantities cause death. On section, all the abdominal organs are found to be stained a deep blue, but the blood and bloodvessels are of normal appearance. This is very well seen in the kidney. Death is caused in such cases from the staining, and also from paralysis of the important nerve centres. There is no trace of serous exudation or of intlam. mation found on section, even in the peritoneal cavity. Rabbits eat the dye, if properly mixed with their food, in large quantities, and without any injurious effects. On section the intestine appears moderately blue stained. The firces of an animal fed on $\mathrm{m}$. $v$. are of an intensely blue colour.

3. Therapeutical and other experiments - I inoculated a rabbit's eye with staphylococcus pyogenes aureus, and thus caused in it a severe hypopion. I then dropped in some m. v. solution, thus causing deep staining of the whole extent of the external wound, and also of the flaky collections of pus in the anterior chamber, and putting a stop to the suppurative process. I have obtained in my clinic most successful results in the treatment of corneal ulcers which had hitherto resisted all the usual remedies, and I am of opinion that the $m$. v. treatment for these cases will supersede that by the galvano-cautery. I have also used it with very good results in a number of other external eye diseases-such as blepharitis, conjunctivitis, phlyctrena, and eczema of the lids. I have also used it with success in internal eye diseases, such as keratitis parenchymatosa, iritis serosa, old-standing cases of choroiditis, and even in one case of sympathetic ophthalmia. I have also had good results in some few surgical cases which have come into my hands, such as varicose ulcers \&c., but of course I have not had nuch opportunity of following up this class of treatment. Another important point is this. Since, as has been shown above, one can inject relatively large quantities into the peritoneal and pleural cavities without injury, and as animals can eat also, without injury, large quantities of the dye, so also is its further application conceivable, and the methyl violet treatment of purulent pleurisy and peritonitis, typhoid and dysenteric ulcers, \&c., is not beyond the bounds of possibility. A slight disadvantage of the surgical application of $\mathrm{m}$. $v$. is that one is liable to stain the fingers and hand blue. This staining is, however, easily removed by alcohol, ammonium sulphide, \&c. The forms in which I have used the substance are those of solutions, pencils, powders, and unguents, and occasionally the pure substance itself. I shall discuss the details as to the employment and dosage of the individual preparations in my second paper.

Birkenhead:

\section{AN OPERATION FOR VESICO-VAGINAL FISTULA THROUGH A SUPRA-PUBIC OPENING IN THE BLADDER. ${ }^{1}$}

By A. F. MCGILL, F.R.C.S., SURGEON TO TIIE LEEDS GENERAL INFIRMARY.

AN operation for vesico-vaginal fistula through a suprapubic wound in the bladder was first suggested by Professor Trendelenberg. I have operated in this way twice. The first case was one of epithelioma, involving the floor of the urethra for its wholelength, as well as part of the anterior vaginal wall and base of the bladder. The opening made in removing the growth was so large that, though I succeeded in bringing the edges of the hole which I had formed into apposition, the wound subsequently gaped, and a large vesico-vaginal fistula resulted. The chief interest in this case lies in the fact that in five weeks, without further treatment, owing to the efficient supra-pubic drainage, the fistula entirely and spontaneously closed. The second case was an ordinary one following labour, in which the operation was perfectly satisfactory.

CASE 1.-E. S-, aged fifty, was admitted into the Leeds Infirmary under my care on Sept. 19th, 1889, suffering from retention of urine. For twenty-seven years she bad taken laudanum (about an ounce and a half daily) Her health was good, and she had no urinary symptoms till five months before adnission; she then noticed that she had difficulty in passing urine, that micturition was accompanied by pain, and that occasionally the urine was tinged with blood. The symptoms gradually increased in severity, till seven days previously she had complete retention; she has not been able to pass any urine, but has been relieved by dribbling overflow. On admission, the bladder was found to be much distended. There was a hard, nodular mass, which extended from the urinary meatus on to the anterio vaginal wall; it involved the floor of the urethra and excended on to the base of the bladder. A female catheter was introduced with some difficulty, and several pints of urine were evacuated. The retention recurred, She was only relieved by frequent catheterism, and this caused much pain and some hæmorrhage. It was consequently determined to operate by removing the growth closing the vesicovaginal fistula thus formed, and leaving a supra-pubic opening. On Sept. 27 th, the pubes having been shaved, the patient, who was a very thin woman, was placed in the inverted position by hanging her by her knees to a crossbar attached to an operating table and supporting her loins by pillows. Her head was placed towards a window, the full light of which consequently fell on the abdomen. The bladder was now distended with twelve ounces of a boracic acid solution. A transverse incision, about half an inch above the pubes and three inches in length, was made; this divided both skin and recti muscles. The bladder was exposed, fixed by a tenaculum and opened transversely; it was fixed to the deep part of the abdominal wall, and thus prevented from falling into the pelvis, by three sutures. The patient was next placed in the lithotomy position, and a duckbill speculum introduced into the vagina. The growth, pressed down by the fingers of an assistant passed through the supra-pubic wound, was removed by scalpel and scissors. It was more extensive than was expected. The lower two-thirds of the circumference of the urethra, as well as part of the base of the bladder, was implicated, and when the removal was complete, an opening, which would easily admit two fingers, had been made. This opening was closed by fine silk sutures passed through the vaginal wall. The edges were with difficulty brought together, and there was much tension on the stitches. The patient was now replaced in the inverted position, and the opening in the mucous membrane of the bladder was closed by fine catgut sutures. Lastly, the supra-pubic wound in the bladder was closed at each end, an opening being left large enough to admit a drainage-tube; the recti muscles were stitched together and the skin wound closed except at the centre. On injecting the bladder, it was found that the opening into the vagina was watertight. The pathological report made it certain that the growth was a squamous epithelioma. The subsequent history was as follows. On examination at the end of a week, it was found that the

1 A paper read at the Medical Society of London, Nov. 3rd, 1890. 